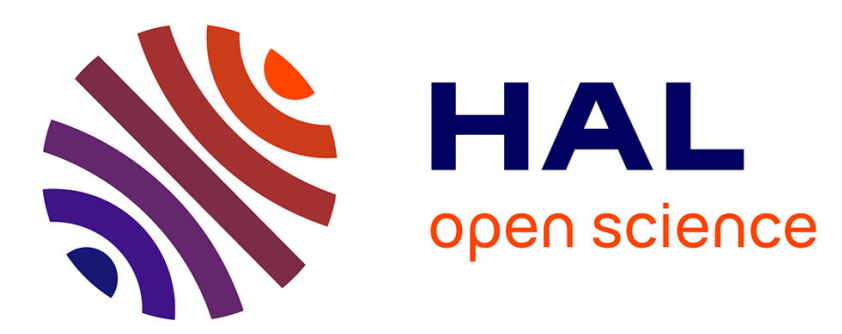

\title{
Effectiveness and safety of topical capsaicin cream in the treatment of chronic soft tissue pain
}

Sigrun Chrubasik, Thomas Weiser, Beate Beime

\section{To cite this version:}

Sigrun Chrubasik, Thomas Weiser, Beate Beime. Effectiveness and safety of topical capsaicin cream in the treatment of chronic soft tissue pain. Phytotherapy Research, 2010, 24 (12), pp.1877. 10.1002/ptr.3335 . hal-00590595

\section{HAL Id: hal-00590595 https://hal.science/hal-00590595}

Submitted on 4 May 2011

HAL is a multi-disciplinary open access archive for the deposit and dissemination of scientific research documents, whether they are published or not. The documents may come from teaching and research institutions in France or abroad, or from public or private research centers.
L'archive ouverte pluridisciplinaire HAL, est destinée au dépôt et à la diffusion de documents scientifiques de niveau recherche, publiés ou non, émanant des établissements d'enseignement et de recherche français ou étrangers, des laboratoires publics ou privés. 


\section{Effectiveness and safety of topical capsaicin cream in the treatment of chronic soft tissue pain}

\begin{tabular}{|c|c|}
\hline Journal: & Phytotherapy Research \\
\hline Manuscript ID: & PTR-10-1074 \\
\hline Wiley - Manuscript type: & Full Paper \\
\hline $\begin{array}{l}\text { Date Submitted by the } \\
\text { Author: }\end{array}$ & 01-Oct-2010 \\
\hline Complete List of Authors: & $\begin{array}{l}\text { Chrubasik, Sigrun; Institute of Forensic Medicine, University of } \\
\text { Freiburg im Breisgau; Sydney University, Herbal Medicines } \\
\text { Research and Education Center } \\
\text { Weiser, Thomas; Boehringer Ingelheim Pharma GmbH \& Co. KG } \\
\text { Beime, Beate; Diapharm Clinical Management GmbH }\end{array}$ \\
\hline Keyword: & $\begin{array}{l}\text { soft tissue pain, low back pain, capsaicin, placebo, clinical trial, } \\
\text { chronic pain }\end{array}$ \\
\hline
\end{tabular}

\section{SCHOLARONE ${ }^{\text {M }}$ \\ Manuscripts}


Effectiveness and safety of topical capsaicin cream in the treatment of chronic soft tissue pain

S. Chrubasik ${ }^{1}$, T. Weiser ${ }^{2}$, B. Beime ${ }^{3}$ for the study group

${ }^{1}$ Institute of Forensic Medicine, University of Freiburg, Albertstr. 9, D 79104 Freiburg

${ }^{3}$ Boehringer Ingelheim Pharma GmbH \& Co. KG, Binger Straße 173, 52216 Ingelheim

${ }^{4}$ Diapharm Oldenburg, Würzburger Straße, D-26121 Oldenburg

Adress of Correspondence

Prof. Dr. Sigrun Chrubasik

Institute of Forensic Medicine

University of Freiburg

Albertstr. 9

D 79104 Freiburg

Germany

\section{Key words}

chronic pain, soft tissue pain, low back pain, capsaicin, placebo, clinical trial 


\begin{abstract}
Topical capsaicin is an established treatment option for various pain conditions. In a randomised double-blind multi-center study, 281 patients suffering from chronic soft tissue pain were treated either with a cream containing capsaicin $0.05 \%$ ("Finalgon $\AA$ CPD Wärmecreme", $n=140)$ or placebo $(n=141)$. Of these, 151 were excluded from the ITT analysis, as they had in addition to their soft-tissue pain, pain of other origin.

The primary outcome measure was a positive treatment response, defined as a pain sum score reduction of $30 \%$ or more. After 3 weeks of treatment, the median pain sum score had decreased by $49 \%$ (capsicum) group and 23\% (placebo) (ITT analysis, $\mathrm{p}=0.0006$ ). The odds ratio of the responders in favour for capsaicin was 4.3 (CI 97.5\% lower limit 1.9, p $<0.0001$ ). Improvements in the secondary efficacy measures confirmed the results. Likewise, all outcome measures had significantly more improved in the capsaicin-treated compared to the placebo-treated chronic back pain sufferers. All patients were included in the safety assessments. More adverse events occurred in the capsicum group $(n=13)$ than in the placebo group $(n=6)$. The capsaicin cream was generally well tolerated.

Our results indicate that capsaicin cream is useful in patients with chronic soft tissue pain and is also efficacious in patients with chronic back pain for which effectiveness was already demonstrated in earlier clinical trials.
\end{abstract}




\section{Introduction}

Among painful musculoskeletal conditions, chronic pain of the soft tissues (muscles, fascia, tendons, ligaments, cartilage, synovium, fibrous capsules, organs, and nerves) is as frequent as symptomatic peripheral osteoarthritis. For both, a prevalence of around $9 \%$ was calculated from a regional community-based study in Italy (Salaffi et al., 2005). Chronic soft tissue pain can be just as debilitating as pain from bony structures. Among the treatment options for chronic musculoskeletal pain, topical NSAIDs have been shown to be as effective as oral NSAIDs (Mason et al., 2004a). Topical capsaicin products have also shown to relieve a variety of pain conditions including osteoarthritis (Cameron et al. 2009a), rheumatoid arthritis (Cameron et al. 2009b) and low back pain (Keitel et al., 2001, Frerick et al., 2003). The rationale behind this effect is the capsaicin interaction with neurotransmitter, substance $\mathrm{P}$ in sensory $\mathrm{C}$ nerve fibres and its interaction with the vanilloid receptor TRPV1, which triggers thermal and inflammatory pain. Repeated capsaicin applications result in desensitization and inactivation of the neurons which trigger pain. Reversible degeneration of epidermal nerve fibres during prolonged capsaicin exposure contributes to the analgesic effect. Nolano and coworkers (1999) have shown that discontinuation of the application of capsaicin after 3 weeks was followed by re-innervation of the epidermis over a 6-week period with a return of all sensations, except cold, to normal levels. This explains why the capsaicin analgesic effect may carry over after the cessation of treatment. Local skin irritation (sensation of warmth and/or pruritus), which is often mild and transient, is common during treatment with capsaicin products and can be easily explained by its mechanism of action, however, systemic adverse events were not observed in any of the clinical trials.

In a meta-analysis of fibromyalgia treatment interventions, a trial with topical capsaicin is listed (Rossy et al., 1999). This pilot study did not find a significant effect. (McCarty et al., 1994). Other information on the use of topical capsaicin in chronic pain caused by soft tissues 
was not available. The aim of this study was therefore to evaluate the efficacy and safety of a proprietary capsaicin cream $0.05 \%$ in the treatment of chronic soft tissue pain.

\section{Methods}

Design of the study: We conducted a randomized, double-blind, placebo-controlled study in 23 outpatient clinics in Germany. The protocol was approved by the Ethics' Committees responsible for the principal investigator and each trial centre. The study was planned and conducted in accordance with the GCP guidance for the European Community and the ICH GCP guidelines which are part of the German Drug Laws. Informed written consent was obtained from all patients.

The test product was "Finalgon ${ }^{\circledR}$ CPD Wärmecreme", of which $100 \mathrm{~g}$ contain $2.2-2.6 \mathrm{~g}$ soft extract of capsici fructus acer (DER 5.5:1 (4-7:1) corresponding to $53 \mathrm{mg}$ capsaicin $(0.05 \%)$. The reference product was a cream, identical in viscosity, colour and fragrance to the test product, containing no active substance. The cream was coloured with chinolin yellow to ensure an optical match with the yellowish colour of the test product. The decision which treatment a suitable patient should receive was made according to a computerized randomisation list. Because a burning or itching sensation might have demasked the blinding, the patients were informed that absence of this effect did not provide a definite information of the group to which the patients had been assigned. Patients were instructed to apply the cream as a thin layer and rub it onto the painful area thrice daily over 3 weeks. The study consisted of a treatment phase of 21 days and a facultative follow-up of 14 days.

Selection of patients: Patients were eligible if all the following criteria were met according to the history of the patients and the physical examination by the physician: age between 18 and 65 years, Caucasian, chronic pain of the soft tissues of the musculoskeletal apparatus, subjective pain at enrolment $\geq 5$ (VAS 0-10, 0 no pain, 10 intolerable pain), ability and 
expressed willingness of the patient to follow the investigator's instructions, i.e. meeting the prerequisites of the study, applying study medication according to the dosage regimen and filling in the questionnaires at the control visits, and granting of written informed consent. Patients were excluded in case of severe co-morbidity, addiction to alcohol or other drugs, pregnancy and lactation, insufficient contraceptive protection, participation in another clinical trial within the last 4 weeks, concomitant psychiatric disorders, a surgical procedure required in the immediate future, inability of the patient to understand the nature, importance and consequences of the study. Special exclusion criteria: muscle rupture, vertebral disk prolapse, spondylolisthesis, spinal canal stenosis, known or clinically proven instability of the spine, spinal fractures, tumours, infections, inflammatory joint conditions, seronegative spondyloarthropathies, osteoporosis as the cause of pain, chronic skin diseases, known hypersensitivity to capsaicin or other ingredients of the cream, anxiety or depressive conditions, $\geq 11$ points of HADS D anxiety score or HADS D depression score. Concomitant analgesic treatments which could interact with the study medication had to be discontinued before enrolment into the study, Antidepressants, anxiolytics or sedatives, surgical procedures or nerve blocks in the treatment area, systemic opioids, oral corticosteroids, transcutaneous electric nerve stimulation had to be stopped for 4 weeks. Topical antirheumatics on any part of the body surface for 14 days,non-steroidal antiinflammatory drugs, all topical medicines used in the painful area for 7 days, and topical corticosteroids, baths with aromatic oils for 3 days,. If antihistaminics or muscle relaxants were taken, the dose had to remain unchanged from 7 days before the start of study until its end. Additional analgesics were not allowed except for a short term for acute pain conditions, such as tooth ache.

Outcome Measures: The Pain Sum Score (VAS 0-10) consisting of average pain within the last 24 hours, the worst pain within the past 3 days and average pain within the last 3 days, 
giving a maximum total score of 30 was assessed at baseline, after one, two, three and five weeks. The primary outcome measure was treatment response, defined as a Pain Sum Score reduction $\geq 30 \%$.

Secondary outcome measures included the VAS pain subscores and the patients' diaries which contained daily records of the following items: pain intensity before the first cream application and at the moment of maximum pain relief using numeric pain scales $(0-10,0$ no pain, 10 intolerable pain), the delay between application of cream and onset and maximum effect, the duration of analgesia and questions on adverse effects (sensation of warmth, itching). Duration of any sick leave since the past visit was also documented after one, two and three weeks as were the efficacy (investigator: excellent, good, adequate, unsatisfactory; patients: free of complaints, symptoms improved, unchanged, worsened) and adverse events (severity, causal relationship, actions taken, outcome), vital parameters (blood pressure, heart rate), skin status, Specific questions concerning the sensations of warmth or pruritus were recorded: Did you experience these sensations during the past week (yes/no)? If so, at how many days (the first day only, up to the second/third day, the whole week, varying)? If so, how long did it last (1-3h, 3-6h, 6-12h, varying)? Intensity of the sensation: Numeric scale (010) and a global safety evaluation (investigator: good (no ADR), moderate (tolerable ADR), unsatisfactory (severe ADR), patients: good, moderate, unsatisfactory) - some of the variables also assessed after 5 weeks.

\section{Statistical Analysis}

A patient was rated as a responder if the percentage change in the pain sum score relative to the admission value was at least $30 \%$, i.e. [enrolment value minus final assessment value] / enrolment value $x 100 \geq 30$. The $30 \%$ decrease was chosen as therapeutically relevant level, following agreement with the study's rheumatological advisers and rheumatologists in independent centres. It is also known that responder rates of $50 \%$ or more can be achieved on 
placebo treatment in pain therapy trials (Moore et al., 1997). In order to set the difference between the two medications as high as could be reasonably assumed, a minimum responder rate of $65 \%$ was expected for the active treatment after 3 weeks, versus a maximum rate of $45 \%$ for placebo. The main target of the study was the confirmative proof of a superior efficacy of capsicum cream compared to placebo cream, the between-group difference of the responder rate - was estimated by means of the odds ratio:

$$
\mathrm{OR}=(\cdot \operatorname{Verum} *(1-\cdot \text { Placebo })) /\left(\cdot \text { Placebo }^{*}(1-\cdot \text { Verum })\right)
$$

This would result in case of equal responder rates of $\mathrm{OR}=1$. The following hypotheses for the odds ratio were established:

The null hypothesis postulated: $\mathrm{OR} \leq 1$ versus an alternative hypothesis of: $\mathrm{OR}>1$. A onesided $97.5 \%$ confidence interval was calculated for the odds ratio. Only the lower limit was calculated because of the hypothesis of the expected superiority of the capsicum cream. If this limit was above 1, a superior efficacy of the capsicum cream could be shown at a $2.5 \% \mathrm{p}$ level.

Before the analysis, the medication groups were tested for homogeneity on enrolment. Twotailed $90 \%$ confidence intervals were calculated for between-group differences quantified by the MANN-WHITNEY statistic or the odds ratio depending on the type of the variable. The following variables entered the test: per protocol collective (yes/no; odds ratio); gender (odds ratio), age, height and weight (MANN-WHITNEY statistics), duration of the disease (MANN-WHITNEY statistics), pre-treatment non-medicinal and analgesic (yes/no, odds ratio), sick leave before enrolment (yes/no, odds ratio), pain subscores and total score (MANN-WHITNEY statistics), duration of treatment until final visit (MANN-WHITNEY statistics), proportion of patients with "very good" to "good" compliance rating (odds ratio). In case of serious between-group differences in one of these variables with a possible impact 
on the medication effect, adequately stratified analyses of the main target parameter were proposed.

The primary efficacy analysis was carried out on all randomised patients who fulfilled the inclusion criteria and who used trial medication at least once (intention-to-treat (ITT) population). Missing values of efficacy variables were replaced according to the method "last observation carried forward". Drop-outs were rated non-responders, unless they stopped treatment because of being free of symptoms, who were rated responders. An according to protocol (PP) analysis was performed on all patients without relevant protocol deviations. The assignment to the PP collective was done for each single case after closing the data base while the study data were still blinded.

We used our previous experience with topical capsaicin (Keitel et al., 2001, Frerick et al., 2003) to form the present hypothesis. The calculation of the sample size of this study was based on an effect size $\mathrm{OR}=2.27$, positioned in the range of moderate superiority (benchmark 2.232 according to Tritschler, 2.477 according to Hasselblad-Hedges) using the program nQuery Advisor ${ }^{\circledR}$ Release 2.0 from Statistical Solutions for Fisher's exact test, the counterpart to the exact confidence interval for the odds ratio. The one-sided test had a risk of 0.025 (power $90 \%$ ) suggesting group sizes of 138 patients. For quality assurance, the data were monitored.

\section{Results}

A total of 282 patients were recruited. One patient from the placebo group was excluded from all analyses because of non-participation in any of the follow-up visits. Table 1 lists the baseline characteristics of the 281 patients who were considered for analysis. The tabulation of all details has been placed on a website (http//www.uniklinikfreiburg.de/rechtsmedizin/live/forschung/phytomedicine/originalartikel.html). Unfortunately, the major part of the patients did not meet the inclusion criteria of chronic soft tissue pain 
only, since 142 patients also suffered from pain due to degenerative spine diseases (chronic back pain) and 9 patients suffered from degenerative diseases of other joints. The ITT population of chronic soft tissue pain consisted therefore of 130 patients ( $n=64$ group capsicum; $\mathrm{n}=66$ group placebo). Seven patients dropped out (capsicum group: $\mathrm{n}=3$ were symptom free shortly after the beginning of treatment, $n=2$ due to insufficient pain relief, $n=1$ refused to continue, possibly because of poor tolerance, one patient of the placebo group was symptom free shortly after the beginning of treatment).

Metric and nominal variables at baseline were comparable between the groups. Ten patients (6 group capsicum, 4 group placebo) had to be excluded from the per protocol analysis because of major protocol violations such as violations of inclusion criteria concerning age, concomitant therapy or concomitant diseases, or poor compliance. The location of the chronic soft tissue pain of the capsicum and placebo groups is presented in Figure 1 and was comparable between the groups as were the baseline pain sum scores and the size of the painful area (Table 1).

Prior non-drug treatments (mainly massages and electrotherapy) had been used by $31.2 \%$ of the capsicum and 19.7 of the placebo patients and prior drug treatment by $23.4 \%$ and $27.3 \%$, respectively. At inclusion, $87.5 \%$ of the capsicum and $93.9 \%$ of the placebo patients were fit for work. 58\% of the ITT population suffered from concomitant diseases (e.g. hypertension, hyperlipidaemia, thyroid disorders) with no relevant difference between the groups. The patients' reliability was assessed as very good or good in about $90 \%$ of the patients in both groups.

After 2 weeks of treatment, the pain sum score had improved significantly more in the capsicum group compared to placebo (Figure 2). There were also more responders (pain sum score improvement $\geq 30$ and $50 \%$ (sensitivity analysis) in the capsicum group compared to placebo (Figure $3 \mathrm{a}$ and $\mathrm{b})$. The corresponding odds ratios $(30 \% / 50 \%$, (CI 97.5\% lower limit) after 1,2 and 3 weeks were $1.0(0.5) / 0.5(0.1), 2.6(1.2 / 1.9(0.8)$ and $4.3(1.9) / 2.5(1.1)$, 
respectively. For the numbers of cases included, the retrospective calculation of the power was $97 \%$ after 3 weeks of treatment. The PP analysis supported the results of the ITT analysis reported here (see webpage). The improvements of the pain subscores were greater in the capsicum group (Table 2) as were the numbers of patients with a decrease by more than 3 points of average and worst pain compared to placebo groups. At the end of the optional 2 weeks follow-up after completion of the 3 weeks treatment, 46 patients of the capsicum group and 47 patients of the placebo group were available for additional evaluation of the pain score. No relevant between group difference was observed after stopping the cream application. At the final evaluation, the efficacy of the capsicum treatment was rated as good to excellent in $59.3 \%$ of the cases compared to $24.3 \%$ for placebo treatment and $21.9 \%$ (capsicum) and 51,5\% (placebo) respectively, of the patients complained of symptoms unchanged or worsened. The diary pain score from before the first daily application of cream decreased from week to week, the decrease was more pronounced in the capsicum group. Capsicum patients documented on more than half of the treatment days an onset of pain relief within the first hour following application of the cream, compared to only $19-23 \%$ under placebo. Only about one quarter of the times these patients reported to have felt no effect compared to $50-70 \%$ under placebo. The absolute numbers of days where the patients reported an analgesic effect were $>70 \%$ in the capsicum group than in the placebo group (< $30 \%$ ). In $80 \%$ of these patients the maximum effect was reached within 2 hours after application. In about $50 \%$ of the patients of the capsicum group, the pain relieving effect persisted for 2-4 hours.

The 142 patients with chronic soft tissue and vertebral pain formed a rather homogenous chronic back pain group. Baseline characteristics are presented in Table 1 and Figure 4. Chronic back pain sufferers showed pain sum score improvements (Figure 5) and percentages of responders (Figure 6) in the same range as chronic soft tissue pain sufferers (Figures 2 and 3). 
The incidence of adverse drug reactions (ADRs) was low. Three patients in the capsicum treated group suffered from ADRs with a definite causal relationship to the trial medication (2.1\%), which led in all 3 patients to premature termination of treatment. The symptoms reported were an unpleasant local heat sensation in 2 patients and pruritus in the other. No other ADRs with possible or probable relationship to the trial medication occurred. During placebo treatment, no ADRs were recorded, related to the trial medication. The 3 patients who dropped out judged the safety of the capsicum cream as "unsatisfactory" at any visit, while there were no other ratings than "good" in the placebo group. Vital parameters remained unaffected during the course of the study.

The incidence of heat sensation was $83.6 \%, 80.0 \%$ and $78.6 \%$ after one, 2 and 3 weeks for the capsicum group and $33.3 \%$ / $32.6 \%$ and $32.6 \%$ for the placebo group, respectively. Pruritus was seen considerably less frequently, occurring in $18.6 \%, 14.3 \%$ and $11.4 \%$ of the capsicum and in $5.0 \%, 4.3 \%$ and $3.5 \%$ of the placebo patients after 1,2 and 3 weeks of treatment. The severity of the heat sensation in the capsicum group was mainly reported as 2 to 6 , versus 2 to 3 in the placebo group. The severity of pruritus was predominantly reported as $\leq 3$ in both groups. In the capsicum group scores $\geq 3$ and were more frequently mentioned at visit 2 than at later assessments.

At admission about $90 \%$ of patients in both treatment groups showed a normal skin type. After one week of cream treatment, $92.1 \%$ (capsicum) and $97.2 \%$ (placebo) of the patients showed no skin irritations. During the 3-week observation period, only two (1.4\%) patients on active treatment and one $(0.7 \%)$ on placebo showed skin irritation. No serious reactions were observed. In general, investigators and patients rated the tolerability of the capsicum cream as good. Investigators and patients rated the safety of the capsicum cream as "good" in $93.6 \%$ and $92.1 \%$ of the patients and that of the placebo cream by $97.2 \%$ (investigators and patients). 


\section{Discussion}

Based on recommendations of meta-analyses and systematic reviews, Kroenke and coworkers (2009) have developed a stepped care approach to the pharmacotherapy of chronic pain. The spectrum of treatments includes: (1) oral analgesics, (2) tricyclic antidepressants or tramadol, (3) in case of neuropathic pain: gabapentin, duloxetine or pregabalin, (4) in case of fibromyalgia: cyclobenzaprine, pregabalin, duloxetine, or milnacipran, (5) in case of localized neuropathic or arthritic pain: topical analgesics (capsaicin, lidocaine, salicylates) and (6) opioids either applied individually or in combination. The domain of topical capsaicin is primarily among those who are unresponsiveness to other treatments. Three to four local applications of capsaicin over at least 3 weeks in case of musculoskeletal pain or 6 weeks in case of neuropathic pain was associated with a significant higher $50 \%$ reduction of pain than placebo (Mason et al., 2004b). Our results are in accordance with the findings of this metaanalysis. For chronic low back pain, the numbers needed to treat (NNTs) for a 30 and 50\% improvement of the pain sum score after 3 weeks of treatment were calculated to be 6 and 5 , respectively (Frerick et al., 2003). Such a result was also achieved with topical or oral NSAIDs in the management of chronic musculoskeletal conditions. Mason and coworkers have calculated a NNT of 5 for a $50 \%$ reduction of pain due to capsaicin in patients with osteoatrthritis (Mason et al., 2004a).

For the treatment of chronic soft tissue pain, we calculated NNTs of 2.9 for $30 \%$ pain sum score reduction and $4.750 \%$ pain sum score reduction. For comparison with data from the literature we also calculated the NNTs for $50 \%$ reduction of average pain within the past 24 hours (NNT 4.1) and worst pain of the past 3 days (NNT 4.4). All these numbers indicate that the proprietary capsaicin cream has a place in the treatment of chronic soft tissue pain as adjunct or as sole treatment. The response to capsaicin in chronic soft tissue pain is as good as that of chronic back pain for which our data gave NNTs of 4.2 (30\% pain sum score reduction) and 3.9 (50\% pain sum score reduction), respectively. 
Retrospective power calculation revealed that the number of patients with chronic soft tissue pain was associated with a power of $97 \%$ (for a number of 281 patients $99.9 \%$ ). Even smaller groups than the 64 patients in the capsicum and 66 patients in the placebo group would have been sufficient to demonstrate the superiority of the capsaicin cream versus placebo. The high power is reassuring as concerns the reliability of the result (type two error $2.5 \%$ ).

In the clinical trials including patients suffering from osteoarthritis so far, $0.025 \%$ capsaicin cream was used. The capsaicin plasters for the treatment of chronic low back pain released 11 (Keitel et al., 2001) and 22 (Frerick et al., 2003) ug capsaicin per square cm over 4 to 8 hours per day. We used a $0.05 \%$ capsaicin cream whereas in the trials for neuropathic pain $0.075 \%$ capsaicin products were employed (Derry et al., 2009). It may well be that a higher capsaicin concentration or a longer treatment period might result in better pain relief.

Oral treatment of chronic pain is associated with a relatively high risk of adverse drug reactions which may itself cause morbidity, mortality and extra costs (Piramohamed et al., 2004). The advantage of topical capsaicin treatment over other pharmacotherapy is the low risk of adverse events and the minor nature of those which ocurred. Except for the local warmth and pruritus sensation no adverse events attributable to the capsaicin cream were observed. In general, local capsaicin adverse events occurred in around one third of patients and would not have occurred if these patients were treated with placebo (Mason et al., 2004b). However, in few patients, the skin irritation lead to withdrawal from treatment, in our study this was $2 \%$.

Since chronic pain conditions often require long-term treatment, future studies should assess the effectiveness and safety for topical capsaicin at least over a few months. Treatment over 3 months is described for various other pain conditions, e.g. post herpes zoster pain, or pain due to diabetic neuropathy (Mason et al., 2004b). Future studies on osteoarthritis or back pain should also consider the recommended response criterion or criteria of the Orthopaedic Societies OMERACT / OARSI (Pham et al., 2003, 2004) for better comparability. This 
composite measure includes improvements of pain, function and patient's global assessment.

Inclusion of a quality of life measure would in addition give information in that important domain.

\section{Ackknowledgement}

The authors sincerely thank Professor Tom Torda/Sydney for his valuable comments prior to submitting the manuscript. 


\section{References}

Cameron M, Blumle A, Gagnier JJ, Little CV, Parsons T, Chrubasik S. 2009a. Evidence of effectiveness of herbal medicinal products in the treatment of arthritis. Part 1: Osteoarthritis. Phytotherapy Res 23:1497-515.

Cameron M, Blumle A, Gagnier JJ, Little CV, Parsons T, Chrubasik S. 2009b. Evidence of effectiveness of herbal medicinal products in the treatment of arthritis. Part 2: Rheumatoid arthritis. Phytotherapy Res 23:1647-62.

Derry S, Lloyd R, Moore RA, McQuay HJ. 2009. Topical capsaicin for chronic neuropathic pain in adults. Cochrane Database Syst Rev (4):CD007393.

Frerick H, Keitel W, Kuhn U, Schmidt S, Bredehorst A, Kuhlmann M. 2003. Topical treatment of chronic low back pain with a capsicum plaster. Pain 106:59-64.

Keitel W, Frerick H, Kuhn U, Schmidt S, Kuhlmann M, Bredehorst A. 2001. Capsicum pain plaster in chronic non-specific low back pain. Arzneim.-Forsch/Drug Research 51:896903.

Kroenke K, Krebs EE, Bair MJ. 2009. Pharmacotherapy of chronic pain: a synthesis of recommendations from systematic reviews. Gen Hosp Psychiatry 31:206-19.

McCarty DJ, Csuka M, McCarthy G, Trotter D. 1994- Treatment of pain due to fibromyalgia with topical capsaicin: A pilot study. Seminars in Arthritis and Rheumatism. 23(6):41-47.

Mason L, Moore RA, Edwards JE, Derry S, McQuay HJ. 2004a. Topical NSAIDs for chronic musculoskeletal pain: systematic review and meta-analysis. BMC Musculoskelet Disord 5:28.

Mason L, Moore RA, Derry S, Edwards JE, McQuay HJ. 2004b. Systematic review of topical capsaicin for the treatment of chronic pain. BMJ 328(7446):991.

Nolano M, Simone DA, Wendelschafer-Crabb G, Johnson T, Hazen E, Kennedy WR. 1999. Topical capsaicin in humans: parallel loss of epidermal nerve fibers and pain sensation. Pain 81:135-45. 
Pham T, Van Der Heijde D, Lassere M, Altman RD, Anderson JJ, Bellamy N, Hochberg M, Simon L, Strand V, Woodworth T, Dougados M; OMERACT-OARSI. 2003. Outcome variables for osteoarthritis clinical trials: The OMERACT-OARSI set of responder criteria. J Rheumato.30:1648-54.

Pham T, van der Heijde D, Altman RD, Anderson JJ, Bellamy N, Hochberg M, Simon L, Strand V, Woodworth T, Dougados M, OMERACT-OARSI initiative. 2004.

Osteoarthritis Research Society International set of responder criteria for osteoarthritis clinical trials revisited. Osteoarthritis Cartilage 12:389-99.

Pirmohamed, M, James, S, Meakin S, Green C, Scott AK, Walley TJ, Farrar K, Park BK, Breckenridge AM. 2004. Adverse drug reactions as cause of admission to hospital: prospective analysis of 18820 patients. BMJ 329:15.

Rossy LA, Buckelew SP, Dorr N, Hagglund KJ, Thayer JF, McIntosh MJ, Hewett JE, Johnson JC. 1999. A meta-analysis of fibromyalgia treatment interventions. Ann Behav Med. 21:180-91.

Salaffi F, De Angelis R, Grassi W; MArche Pain Prevalence; INvestigation Group (MAPPING) study. 2005. Prevalence of musculoskeletal conditions in an Italian population sample: results of a regional community-based study. I. The MAPPING study. Clin Exp Rheumatol. 23:819-28. 
Table 2

Relative pain subscore improvements (\%) (Median, 25/75 IQ) ${ }^{*} p<0.05,{ }^{* *} p<0.01,{ }^{* * *} p<0.001$

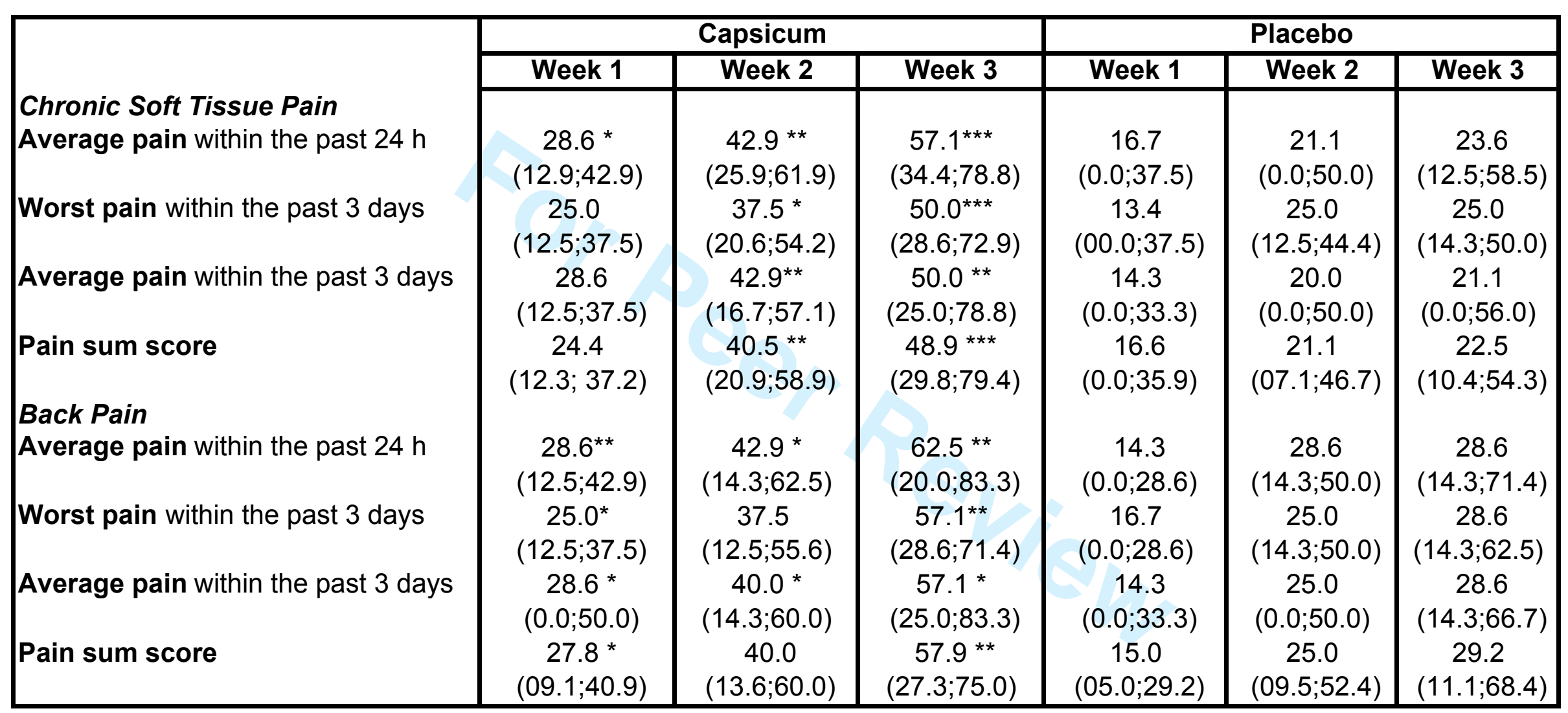


Table 1

Biometrical data, duration of complaints, size of painful area and baseline pain sum score of all patients and those suffering from chronic soft tissue pain and back pain in the capsicum and placebo groups

(mean \pm standard deviation)

\begin{tabular}{|c|c|c|c|c|c|c|}
\hline & \multicolumn{2}{|c|}{ All patients } & \multicolumn{2}{|c|}{ Chronic Soft Tissue Pain } & \multicolumn{2}{|c|}{ Chronic Back Pain } \\
\hline & Capsicum & Placebo & Capsicum & Placebo & Capsicum & Placebo \\
\hline & $n=140$ & $n=141$ & $n=64$ & $n=66$ & $\mathrm{n}=71$ & $n=71$ \\
\hline Age (ys) & $48.6 \pm 13$ & $48.9 \pm 13$ & $48.9 \pm 14$ & $49.9 \pm 14$ & $48.1 \pm 12.6$ & $48.4 \pm 12.1$ \\
\hline Height (cm) & $170 \pm 9$ & $168 \pm 8$ & $170 \pm 9.4$ & $167 \pm 7.8$ & $170 \pm 8.3$ & $168 \pm 8$ \\
\hline Weight (kg) & $76.3 \pm 15.6$ & $72.6 \pm 13.7$ & $77.2 \pm 17.3$ & $72.9 \pm 14.7$ & $76.1 \pm 14.1$ & $72.4 \pm 13.1$ \\
\hline Sex (males) & 59 & 48 & 32 & 22 & 25 & 24 \\
\hline Duration of complaints & & & & & & \\
\hline$<2$ weeks & 8 & 8 & 6 & 2 & 2 & 6 \\
\hline 1 month & 27 & 27 & 13 & 17 & 14 & 9 \\
\hline 2-3 months & 23 & 20 & 12 & 13 & 10 & 7 \\
\hline 4-12 months & 21 & 25 & 8 & 13 & 11 & 10 \\
\hline$>1$ year & 61 & 61 & 25 & 21 & 34 & 39 \\
\hline $\begin{array}{l}\text { Size of painful area }\left(\mathrm{cm}^{2}\right) \\
\text { Pain Sum Score }\end{array}$ & $300 \pm 309$ & $310 \pm 325$ & $\begin{array}{l}269 \pm 236 \\
20.8 \pm 3.1\end{array}$ & $\begin{array}{l}272 \pm 304 \\
21.2 \pm 3.1\end{array}$ & $\begin{array}{l}342 \pm 365 \\
20.5 \pm 2.4\end{array}$ & $\begin{array}{l}358 \pm 344 \\
20.5 \pm 2.6\end{array}$ \\
\hline
\end{tabular}




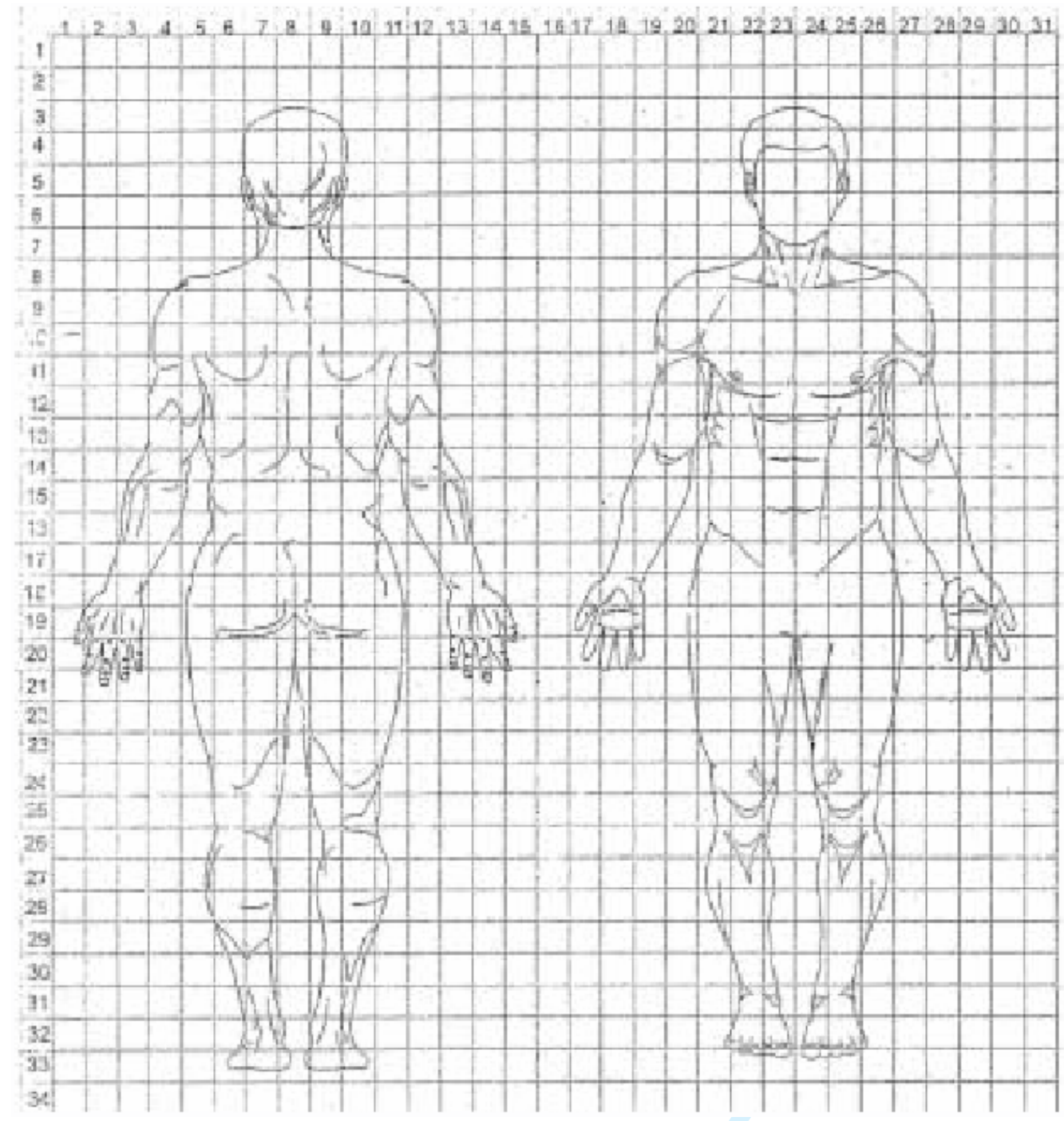

Figure 1a

Body Diagram 


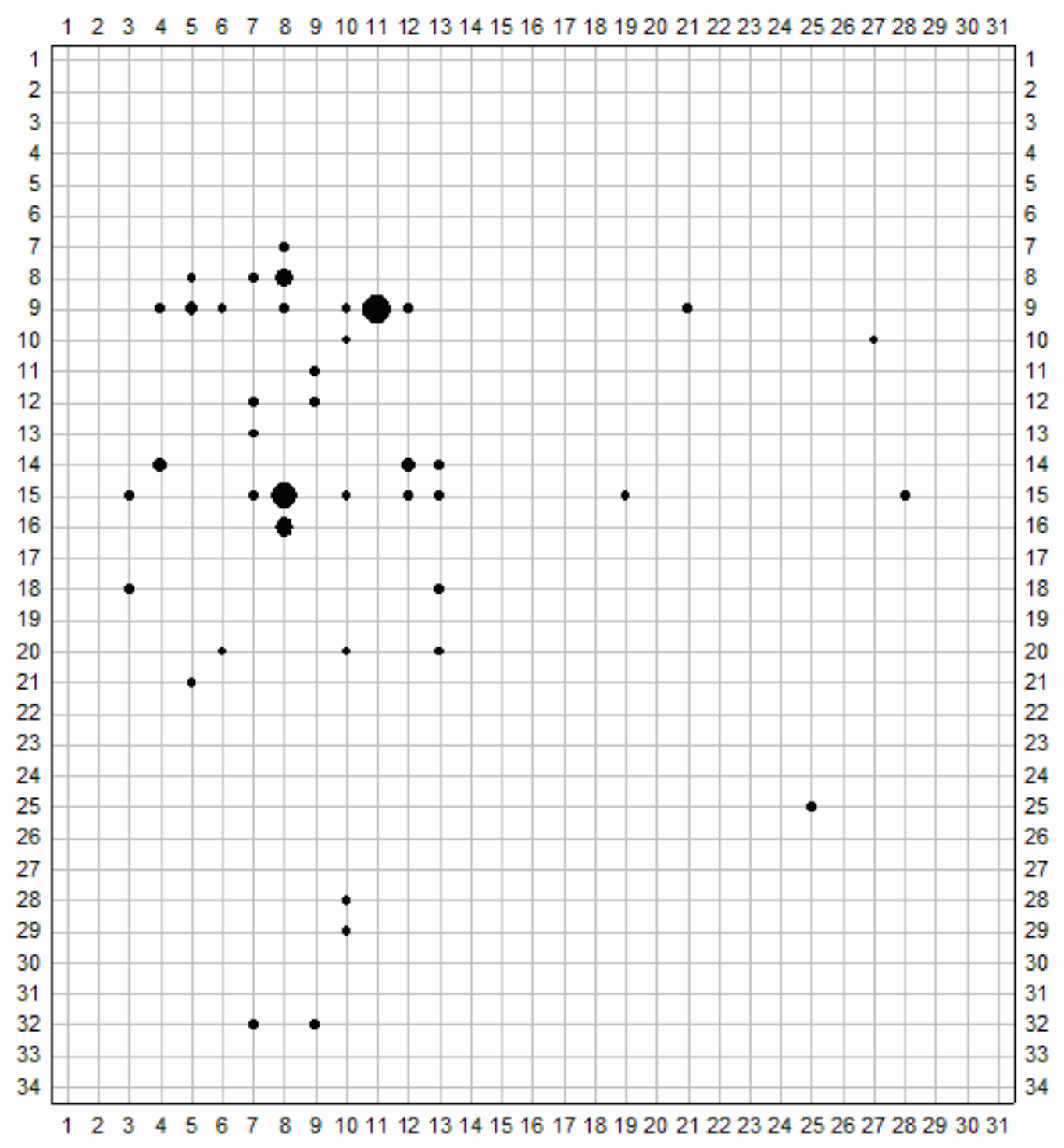

Figure $1 b$

Body diagram of the patients suffering from chronic soft tissue pain in the capsicum group 


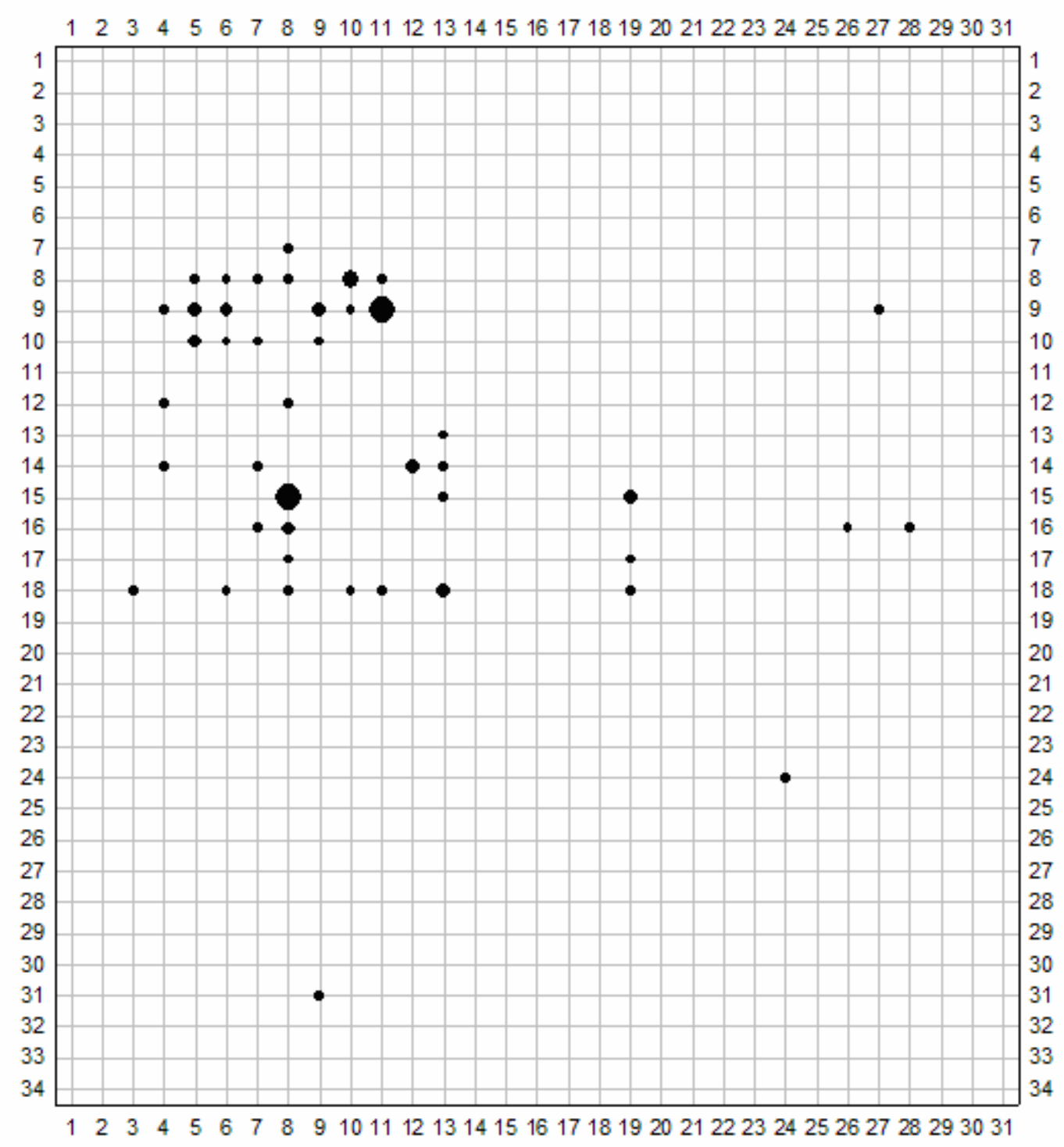

Figure 1c

Body diagram of the patients suffering from chronic soft tissue pain in the placebo group 


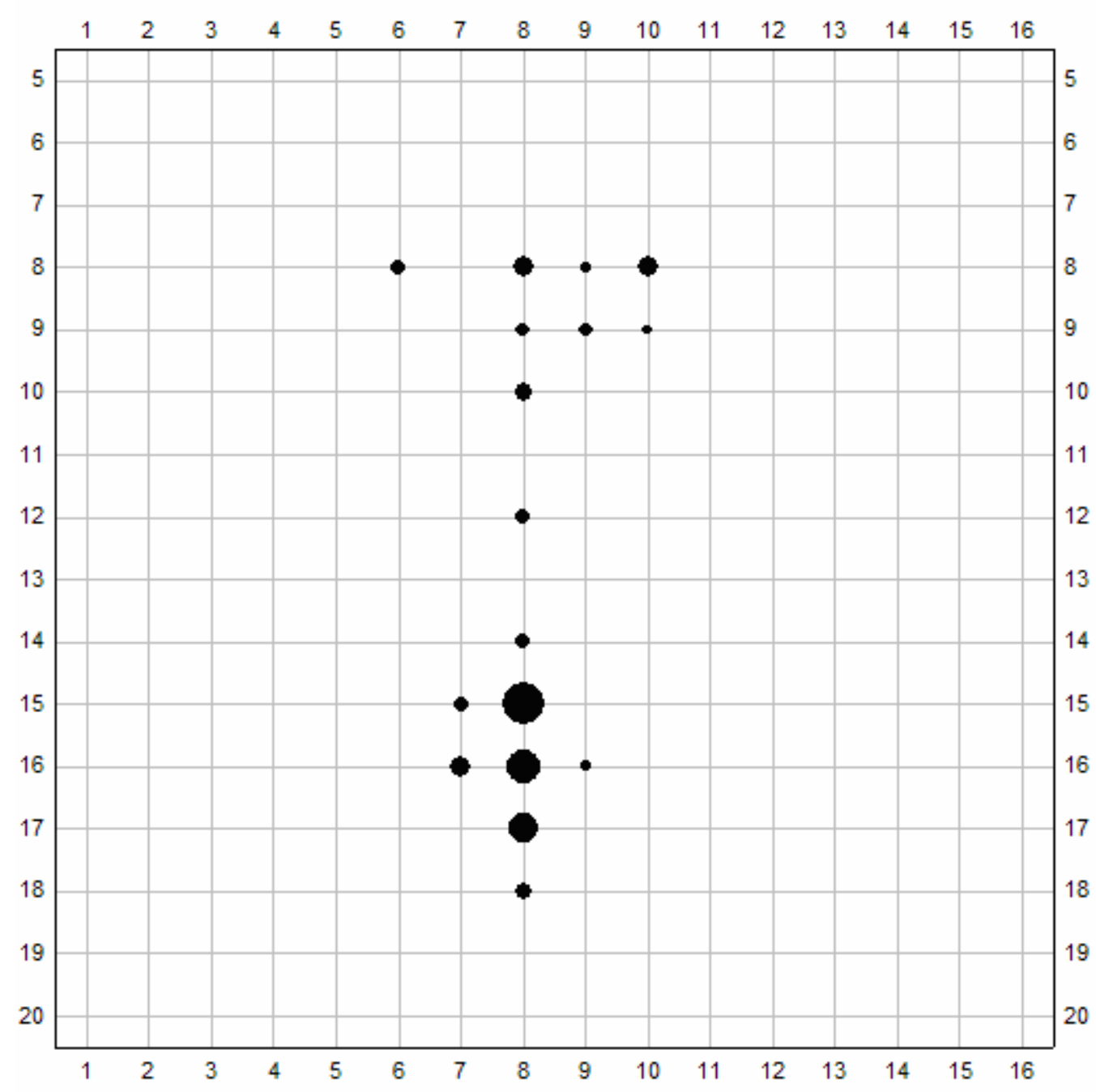

Figure 4 a

Body diagram of the patients suffering from chronic back pain in the capsicum group 


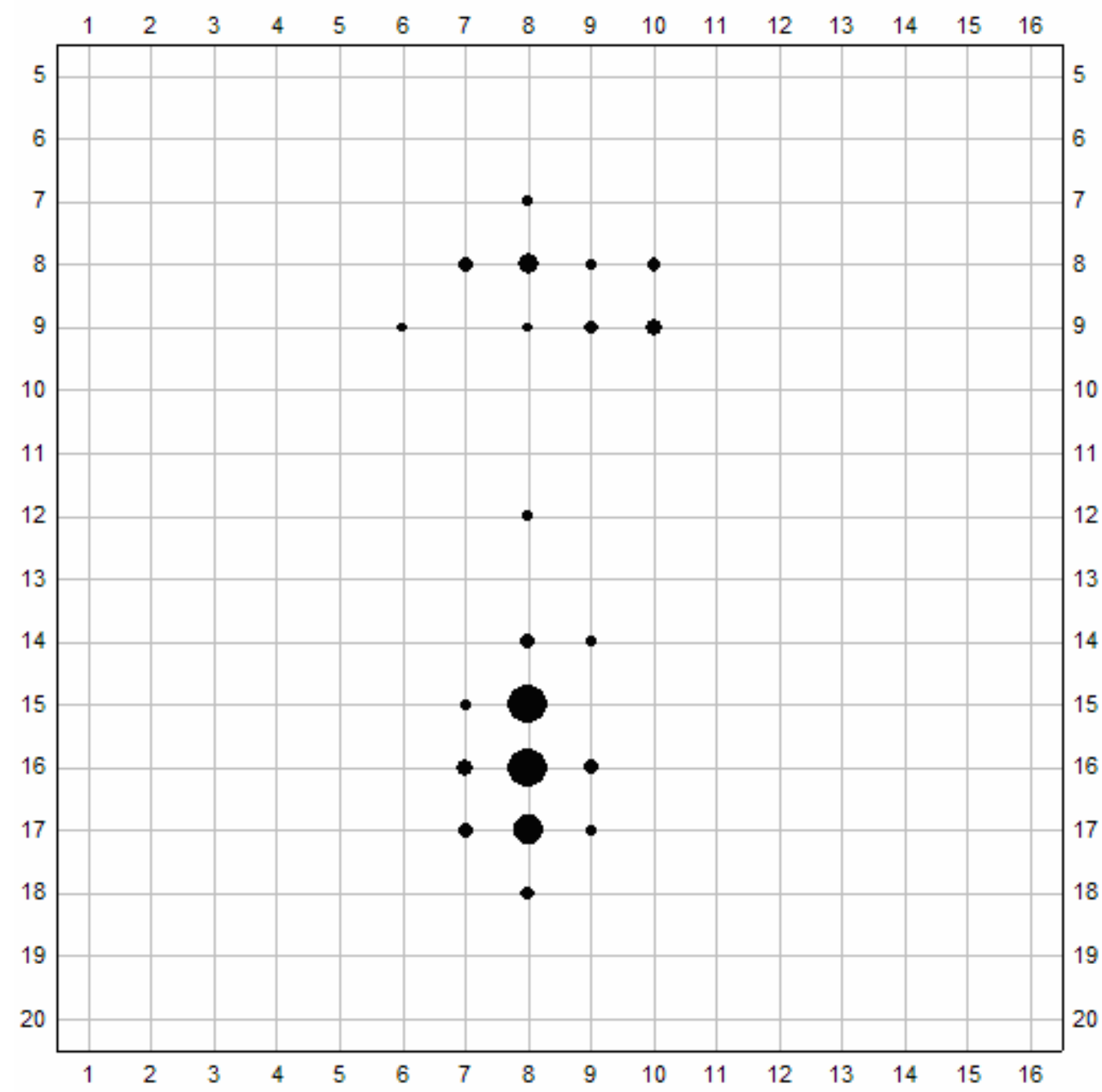

Figure $4 \mathrm{~b}$

Body diagram of the patients suffering from chronic back pain in the placebo group 


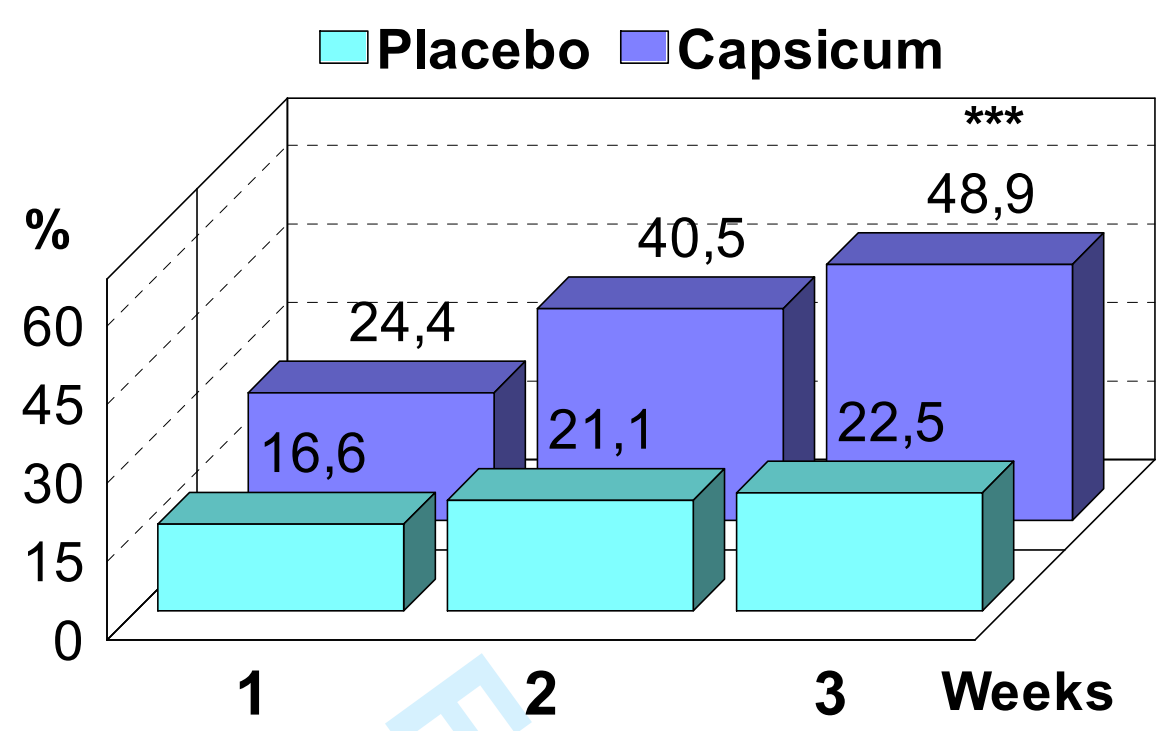

Figure 2

Median relative pain sum score improvement (\%) in the patients suffering from chronic soft tissue pain (ITT analysis)

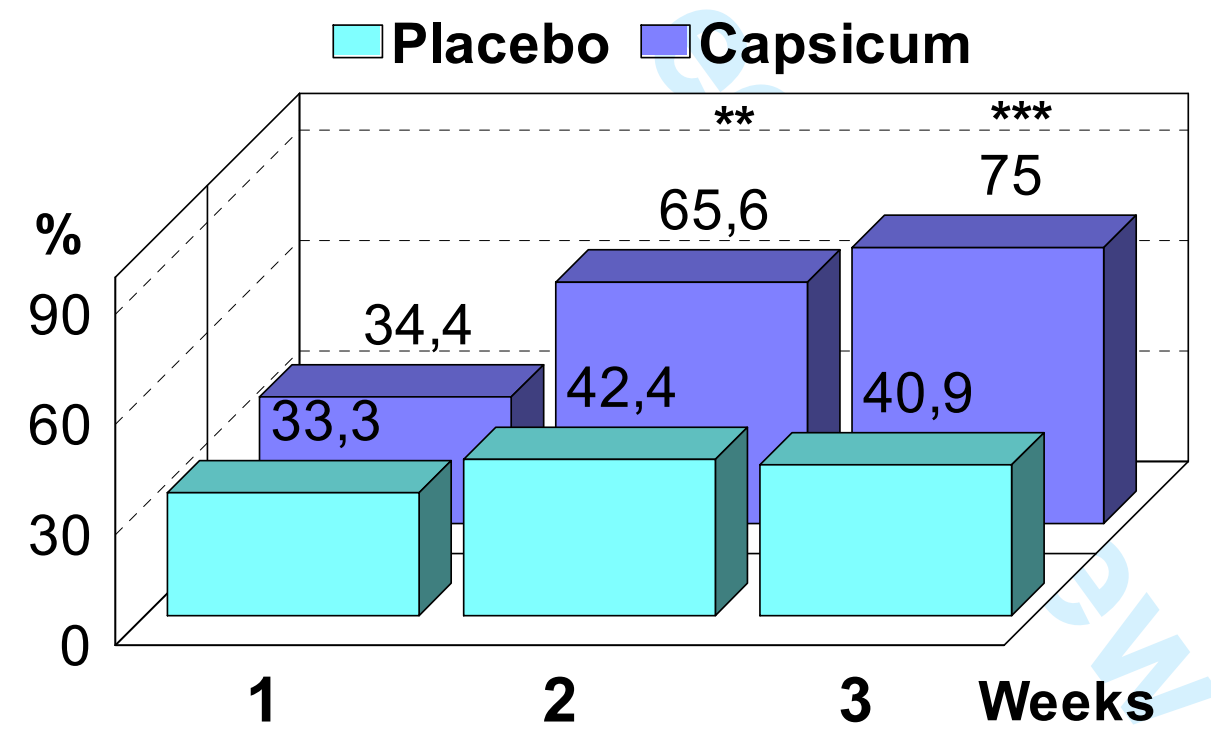

Figure 3a

Chronic soft tissue pain responders ( $\geq 30 \%$ Pain Sum Score improvement) 


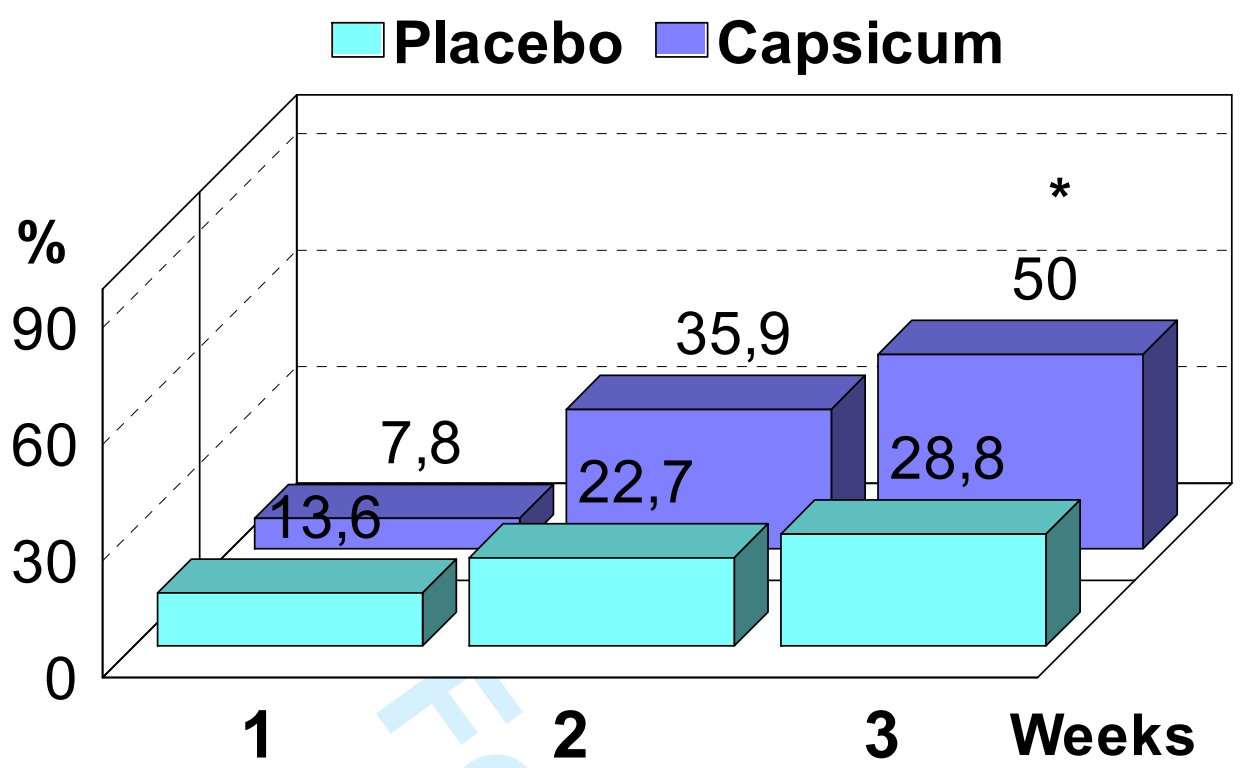

Figure $3 b$

Chronic soft tissue pain responders ( $\geq 50 \%$ Pain Sum Score improvement)

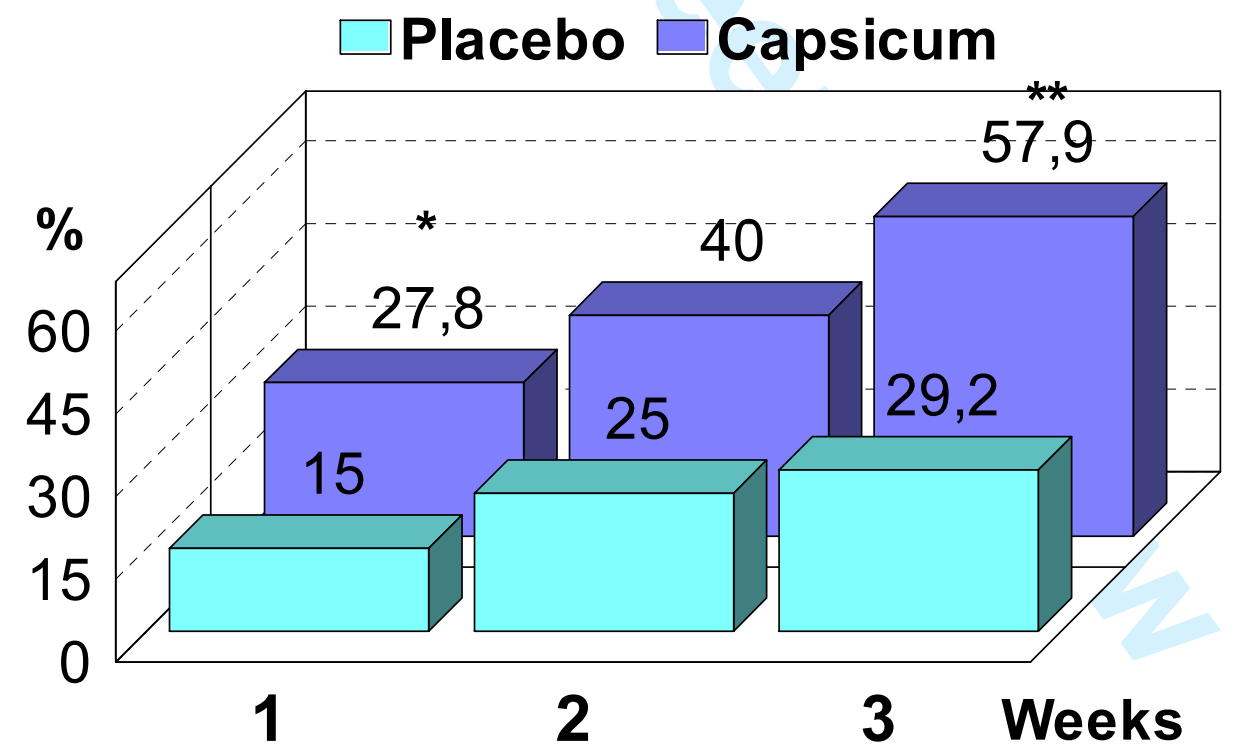

Figure 5

Median relative pain sum score improvement (\%) in the patients suffering from chronic back pain (ITT analysis) 


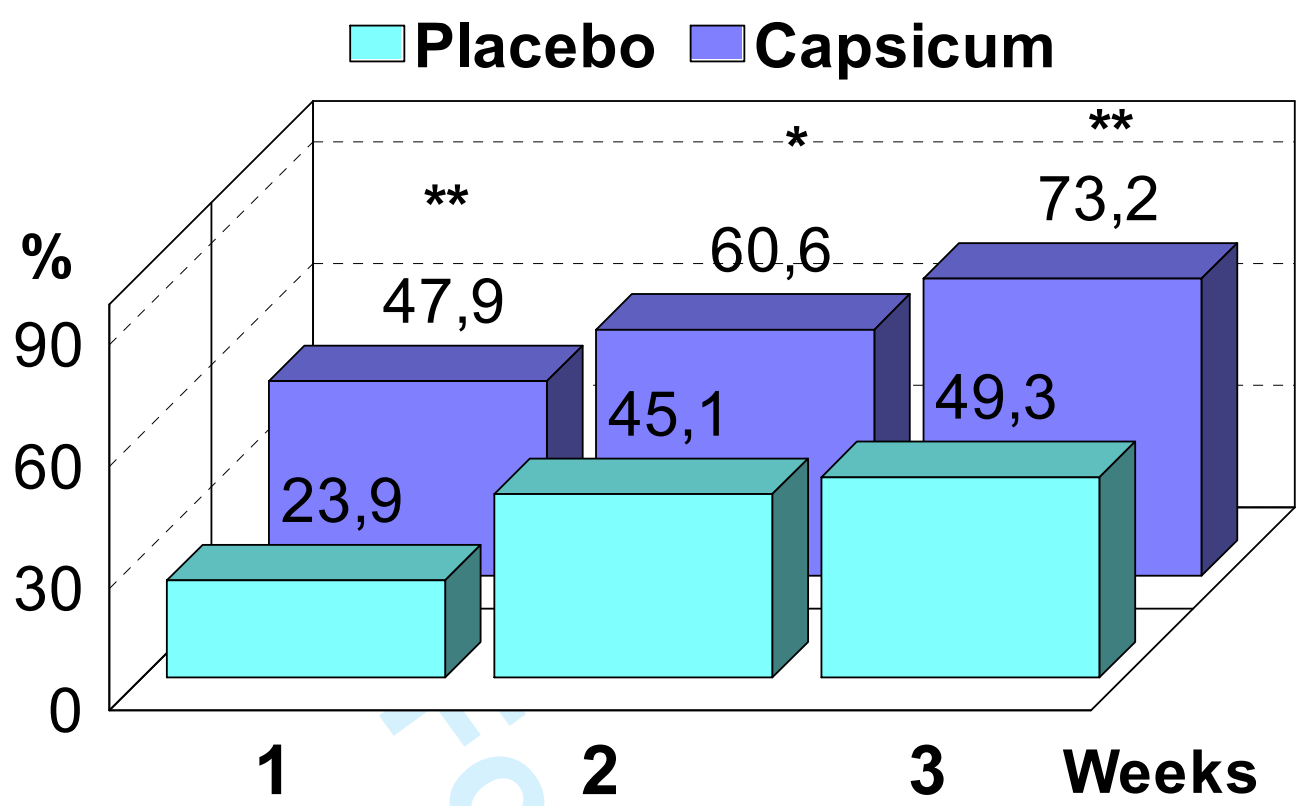

Figure 6a

Chronic back pain responders ( $\geq 30 \%$ Pain Sum Score improvement)

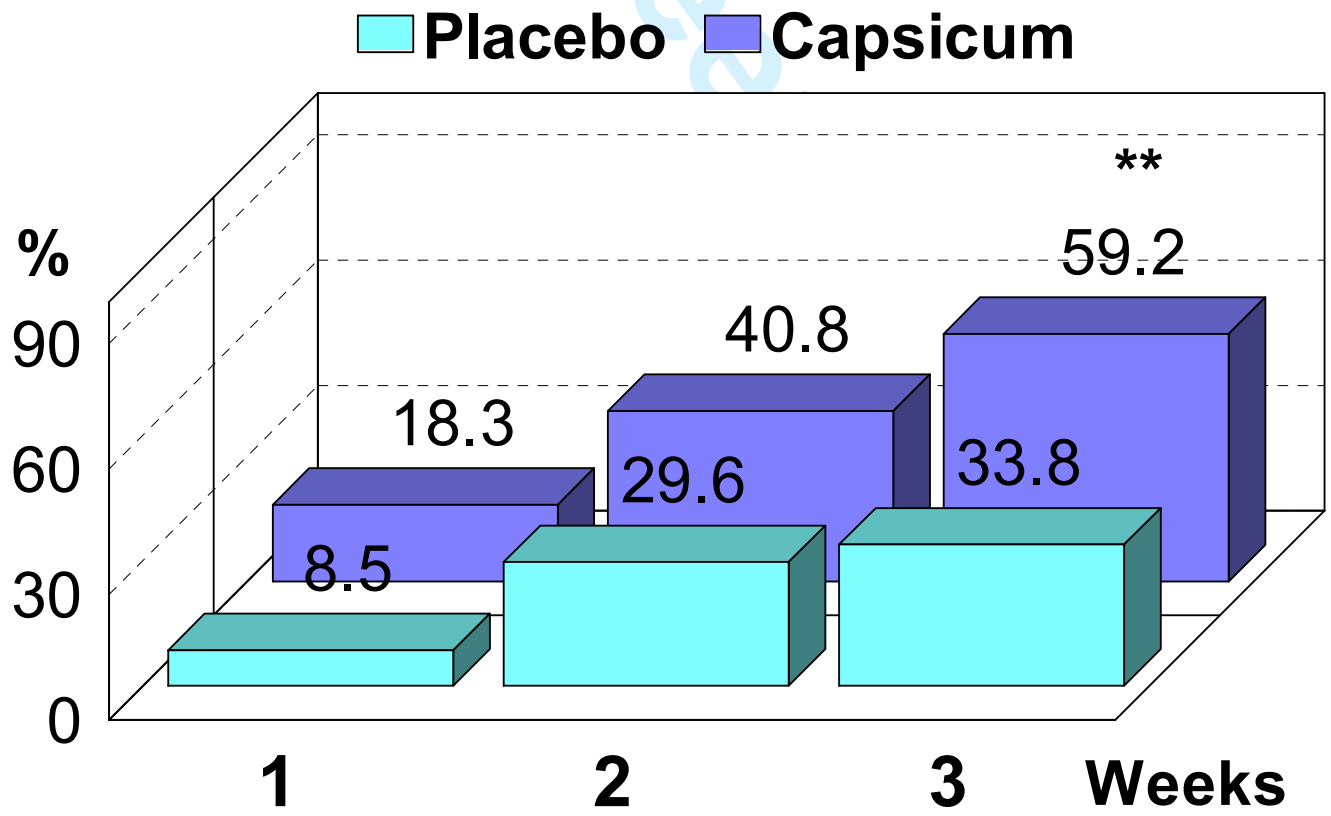

Figure 6b

Chronic back pain responders ( $\geq 50 \%$ Pain Sum Score improvement) 